\title{
Re-use of commercial microfluidics chips for DNA, RNA, and protein electrophoresis
}

\author{
Thi Nguyen ${ }^{\dagger}$, Sukyoung Kwak ${ }^{\dagger}$, and Steven J. Karpowicz \\ Department of Biology, University of Central Oklahoma, Edmond, OK \\ †T.N. and S.K. contributed equally to this work
}

BioTechniques 57:267-271 (November 2014) doi 10.2144/000114232

Keywords: microfluidics; chip; electrophoresis; Bioanalyzer

Supplementary material for this article is available at www.BioTechniques.com/article/114232.

Microfluidics chip technology is a powerful and convenient alternative to agarose gels and PAGE, but costs can be high due to certain chips being non-reusable. Here we describe a method to regenerate, re-use, and store Agilent DNA, RNA, and protein electrophoresis chips designed for use in the Bioanalyzer 2100. By washing the sample wells and displacing the old gel matrix with new gel-dye mix, we have run samples on the same chip up to ten times with negligible loss of signal quality. Chips whose wells were loaded with buffer or water were stored successfully for one week before re-use.

Gel electrophoresis is a common method for analyzing the quality and quantity of DNA, RNA, and protein (1). Recently, microfluidics chip technology has emerged as an alternative to standard agarose and PAGE because it uses less sample, is less time consuming, and yields digital data at a higher resolution scale compared with running and photographing a gel (2-4). However, the benefits of a chip are balanced against increased cost and reduced precision in differentiation between samples of similar molecular weights, when compared with a high percentage polyacrylamide gel. Microfluidics chips require only $1 \mu$ of sample at concentrations as low as the $\mathrm{ng} / \mu \mathrm{l}$ or $\mathrm{pg} / \mu \mathrm{l}$ range (Agilent Technologies Manuals, reference numbers G2938-90024, G2938-90034, and
G2938-90063). The sample is passed through micrometer-scale capillaries that are etched into a glass surface and filled with a gel matrix. As sample molecules approach a waste well that serves as the positive pole, dyes mixed within the gel matrix bind the molecules of interest, are excited by a laser, and fluoresce, thus providing the means of sample detection (2).

However, the need for specialized equipment and a high cost-per-run compared with standard gels serve as barriers to the adoption of microfluidics for electrophoresis. Because the chip quantity is often the limiting factor in a commercial kit, one way to decrease cost-per-run is to use the same chip more than once. Previous research has shown the potential to clean DNA chips by removing the gel matrix and washing the capillaries with $\mathrm{NaOH}$, but inadequate rinses will lead to sample degradation in a subsequent chip run (5).

Here, we investigated a simple method for the re-use of DNA, RNA, and protein electrophoresis chips for the Agilent Bioanalyzer 2100 (Agilent, Santa Clara, CA). We developed protocols by which the chips may be utilized during multiple runs over several days, with equivalent data quality output.

Agilent RNA 6000 Nano chips were tested with total RNA isolated from human liver carcinoma Huh7 cells (6) via phenol/chloroform extraction. The first run of an RNA chip followed the manufacturer's instructions. Upon run completion, the chip was inverted and vigorously knocked against a paper towel on a countertop to remove the liquid from the wells. All wells were rinsed with $20 \mu \mathrm{L}$ of $1 \times$ Tris-AcetateEDTA (TAE) buffer. The chip was vortexed, and the liquid was knocked out again.

A wash run is necessary for RNA chips to remove contaminants from the old gel matrix. We surmised that a component of the gel or loading buffer, possibly formaldehyde, accumulates in the capillaries and alters the voltage in subsequent runs of the chip if not fully eluted. The wash run was performed by adding gel-dye mix to the three gel wells (marked $\mathrm{G}$ and $\mathrm{G}$ ) and TAE buffer into the sample and ladder wells. The chip was vortexed and placed in the Bioanalyzer. No sample peaks were observed in the wash run results (Figure 1), indicating that residual sample in the wells was removed by the earlier TAE rinse. After completion of the wash run, the liquid in the wells was discarded as before. At this point, a chip could be re-used immediately or stored for later use.

To re-use the chip, it was primed with new gel-dye mix added to the gel loading well (G). Air pressure from the loading plunger resulted in the new gel-dye mix physically displacing the old gel, which escaped to the fifteen

\section{METHOD SUMMARY}

Here we show that used Agilent microfluidics chips may be stored and re-used multiple times. The wells of chips for RNA may be cleaned with a blank run of $1 \times$ Tris-acetate-EDTA (TAE) buffer, followed by replacement of the gel matrix with new gel. Microfluidics chips for DNA and proteins require only rinsing of the wells and replacement of the gel matrix. 
TAKE ACTION AGAINST

\section{CONTAMINATION WITH \\ QMI $^{\circledR}$ SAFE-SEPTUM}

Sample, Inoculate Or Add Nutrients

To Your Bioreactor Aseptically !

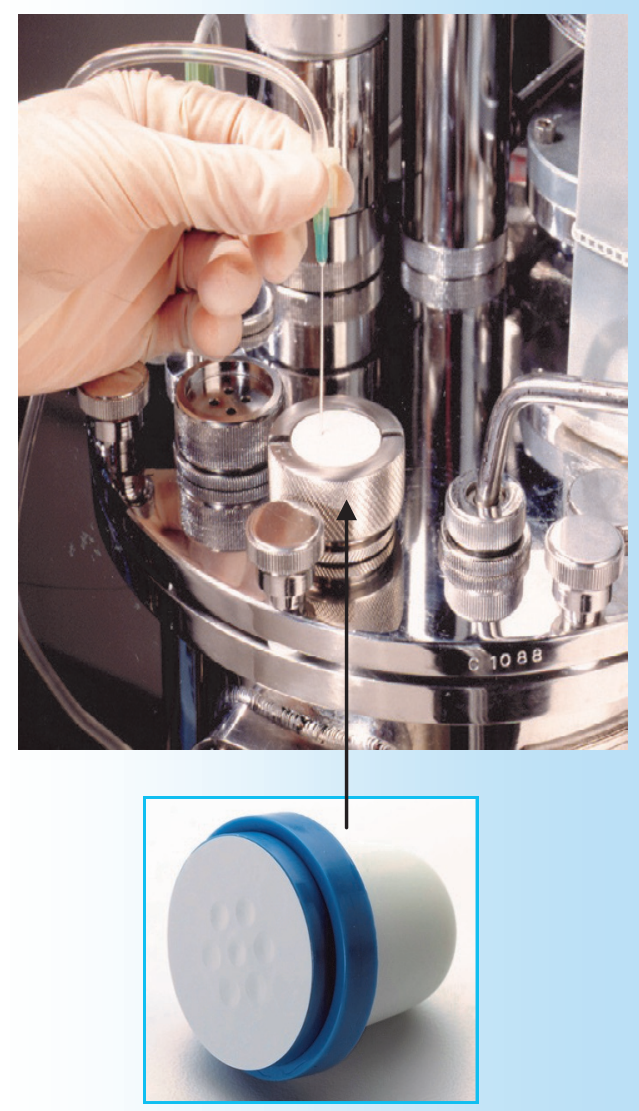

The QMI Safe-Septum is:

- Aseptic

- Pressure \& Temperature Safe

- Pre-Sterilized

- Easy To Retrofit

- Validated

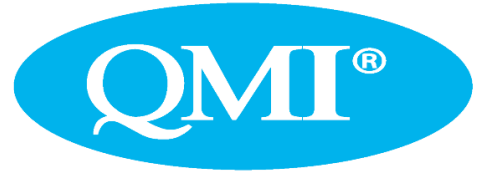

651-501-2337

Email: info@qmisystems.com

www.qmisystems.com open wells. The remaining gel-dye mix still in the loading well was temporarily removed by pipette, and all wells were rinsed with TAE buffer to wash away the old, displaced gel matrix. The wells were emptied by inverting and knocking the chip against a paper towel. To conserve reagents, the removed gel-dye mix in the pipette tip then was returned to the gel loading well. The two waste wells (G), sample wells, and ladder well were loaded as normal. The chip was vortexed, then placed in the Bioanalyzer. If a wash run is not performed or bubbles are introduced into the sample wells or gel-filled capillaries by pipetting error, then the Bioanalyzer software will not detect the presence of the RNA chip.

To store a chip, the rinsed wells were filled with $20 \mu \mathrm{L} 1 \times$ TAE. The gel in the chip's capillaries was not replaced before storage, as fresh gel was added immediately before the next use. The chip was wrapped in Parafilm (Bemis NA, Neenah, WI) and stored at $4^{\circ} \mathrm{C}$ to prevent evaporation, as gel dehydration prevents chip re-use. When intended to be used again, the TAE was removed from the wells, and the old gel in the capillaries was displaced by new gel-dye mix as described above. This protocol provided consistent results in 4 consecutive runs within 1 day (Figure 1A), 5 non-consecutive runs over 2 weeks (Figure 1B), and 10 runs over several days. The observed length of the rRNA was consistent among the 12 sample wells (Table 1). Errors in pipetting the $1 \mu \mathrm{L}$ sample and ladder volumes led to greater variance in quantitation, but values were still within the manufacturer's expected accuracy range.

Agilent DNA 7500 chips were tested using a $1 \mathrm{~kb}$ DNA ladder (ThermoFisher Scientific, Waltham, MA) in 12 twelve sample wells. Re-use and storage of a DNA chip was done following the same protocol as for an RNA chip, including the well rinses, except that the wash run between sample runs is not necessary and has no effect on the results (data not shown). The chips were run successfully over 2 weeks and up to 10 times, with results within the manufacturer's accuracy limits (Table 1).

Agilent Protein 80 chips were tested with a combined sample of BSA, calf intestinal alkaline phosphatase (CIP), and $\mathrm{Bam} \mathrm{HI}$ restriction endonuclease (New England Biolabs, Ipswich, MA) that was heat denatured under reducing conditions. After the standard first run, the chip was prepared for re-use or stored according to the protocol for the DNA chips, except TAE buffer was replaced with ultra-pure water. No wash run is necessary to clean the old gel matrix before it is displaced with new gel-dye mix. Peak sizing results were consistent with the RNA and DNA chips (Figure 2A), but quantitation reproducibility was not as precise, even on the first run of a chip
A

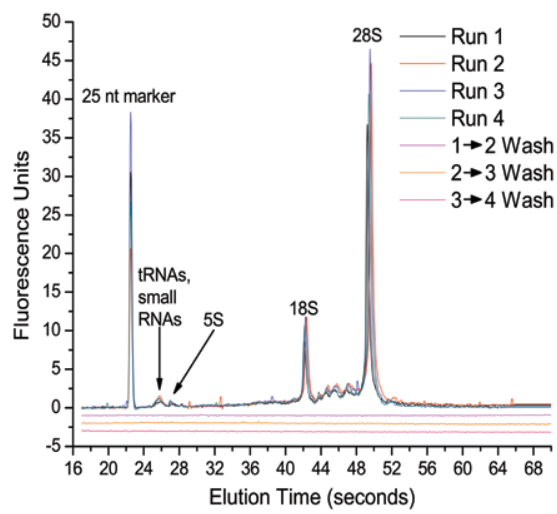

B

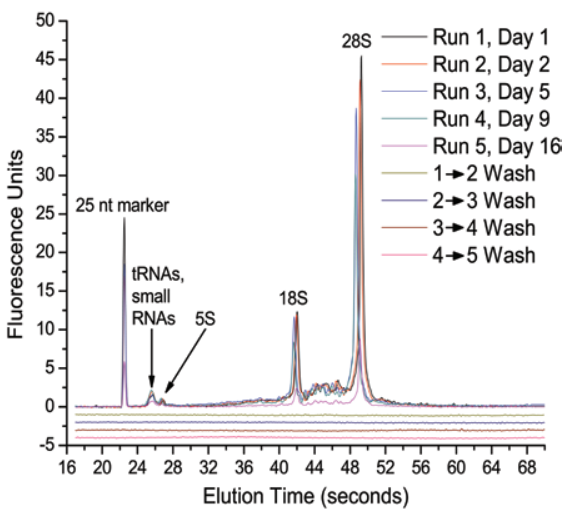

Figure 1. Electropherogram traces from multiple runs of an Agilent RNA Nano chip. (A) An RNA 6000 Nano chip was run consecutively in one day with intermediate wash runs between runs of sample. A representative trace from 1 of the 12 sample wells is shown per run. The wash run traces have been displaced from $y=0$ for clarity. Note that the small, random peaks in runs 2 and 3 result from physical vibrations disturbing the Bioanalyzer. (B) An RNA Nano chip was stored and run non-consecutively over a 2 week period. Note that the weaker signal on run 5 resulted from degraded, light-exposed dye. 
A

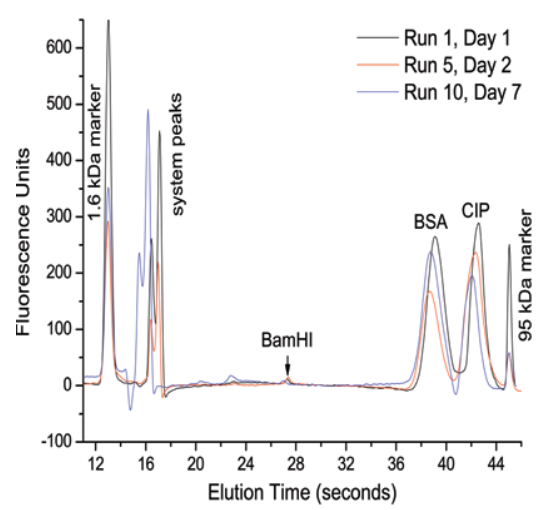

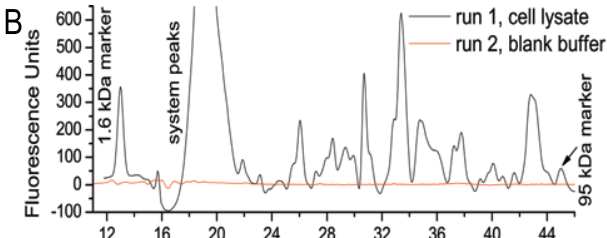

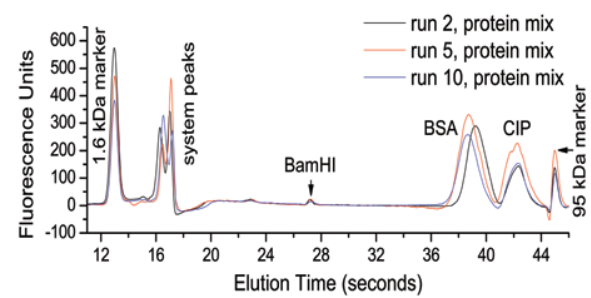

Figure 2. Electropherogram traces from multiple runs of an Agilent Protein $80 \mathrm{chip}$. (A) A Protein 80 chip was stored and run non-consecutively over 7 days. A representative trace from 1 of the 12 sample wells is shown for select runs. Note that the elution time for both samples and ladder can differ slightly between chip runs. (B) Whole cell lysate from Huh7 cells was run on a chip, followed by a run with either blank buffer (upper panel) or BamHI/BSA/CIP sample (lower panel). All subsequent runs used BamHI/BSA/CIP as sample. CIP: calf intestinal alkaline phosphatase.

(Table 1). The Bioanalyzer's analysis software applies baseline correction by default, which is known to affect quantitation accuracy (Agilent Technologies Manual, reference number G293890063). The BSA ran at an apparent weight of $62 \mathrm{kDa}$, which is indicative of 2-(N-morpholino)-ethanesulfonic acidcontaining running buffer.

Whole cell protein lysate was also run on a Protein 80 chip. The chip was prepared for re-use, then the threeprotein sample from above was run. The result was indistinguishable from other uses of the chip with the three-protein sample (Figure 2B).

All three chips may be re-used after a poor result in any or all wells due to degraded quality of sample or reagent. A definitive limit for the number of re-uses was not observed, but individual chips were not tested formally beyond 10 successful runs with loaded sample.

Variants of the Agilent RNA, DNA, and Protein chip classes detect samples of different sizes or concentrations. The variants achieve these ends by altering the details of the sample run through the use of different voltages, marker and ladder sizes, and dye-sample binding methods (http://www.biotechniques. com/BiotechniquesJournal/2010/ September/Therapeutic-proteinanalysis-with-the-Agilent-2100-Bioanalyzer/biotechniques-302535.html). While only one variant from each chip class was tested here, we do not expect that these features would have a substantial effect on the ability of the gel to be replaced and the chip re-run according to our protocol.

In summary, chips may be successfully re-used with no significant alteration in the observed results. An Agilent chip that is run once and discarded, as per standard procedures, costs about $\$ 25$, of which half that cost is for the chip and half for reagents. Following our protocol to re-use a chip 10 times can reduce the cost-per-run by $\sim 40 \%$. Detailed method protocols can be found in the Supplementary Material.

\section{Author contributions}

T.N., S.K., and S.J.K. performed the experiments, analyzed the data, and wrote the manuscript. S.J.K. conceived and designed the study and edited the manuscript.

\section{Acknowledgments}

The authors thank Hari Kotturi for providing the Huh7 cells. This project was financially supported by the College of Mathematics and Science at the University of Central Oklahoma. S.K. was supported by a student research grant from the Office of Research and Grants at the University of Central Oklahoma.

\section{VERSAAATILE IN NEUROSCIENCE

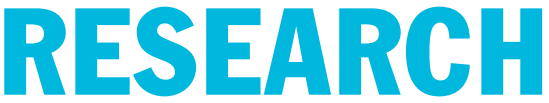

Triple A Polyclonals are all approved in IHC, with images available from several tissues of the central nervous system. The versatility of these antibodies makes them suitable to use in both human and rodent samples. The advanced antigen design, together with antigen based affinity purification, provide you with the most specific, reliable and versatile antibodies available.

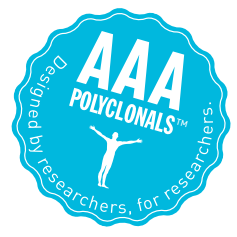

Triple A Polyclonals.

Designed by researchers for researchers

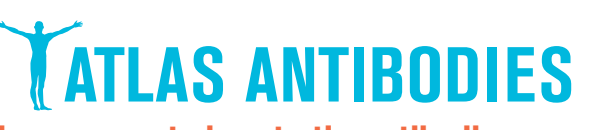


Table 1. Reproducibility of sample peaks and concentrations across chip re-uses.

\begin{tabular}{|c|c|c|c|c|c|c|c|}
\hline \multirow[t]{2}{*}{ Chip } & \multirow[t]{2}{*}{ Peak } & \multicolumn{3}{|c|}{ Range of sizing reproducibility ( \pm CV\%) } & \multicolumn{3}{|c|}{ Range of quantitation reproducibility ( $\pm \mathrm{CV} \%$ ) } \\
\hline & & Min. & Median & Max. & Min. & Median & Max. \\
\hline \multirow[t]{3}{*}{ Agilent RNA Nano } & $25-6000$ nucleotides & \multicolumn{3}{|c|}{ Not provided (Accuracy not provided) } & \multicolumn{3}{|c|}{10 (20 Accuracy) } \\
\hline & 3850 nucleotides (28S) & 0.6 & 1.4 & 2.1 & 2.9 & 5.2 & 8.6 \\
\hline & RIN: 8.5 & 0.6 & 1.3 & 4.5 & & & \\
\hline \multirow[t]{8}{*}{ Agilent DNA 7500} & $100-7500 \mathrm{bp}$ & \multicolumn{3}{|c|}{5 (10 Accuracy) } & \multicolumn{3}{|c|}{ 5-10 (20 Accuracy) } \\
\hline & $517 \mathrm{bp}$ & 0.3 & 1.1 & 2.8 & 2.2 & 6.0 & 12.4 \\
\hline & 806 bp & 0.3 & 1.3 & 2.6 & 2.4 & 5.4 & 13.0 \\
\hline & $1121 \mathrm{bp}$ & 1.1 & 1.6 & 3.9 & 2.2 & 5.0 & 14.2 \\
\hline & $1786 \mathrm{bp}$ & 1.0 & 1.8 & 3.7 & 2.4 & 3.9 & 10.8 \\
\hline & $2332 \mathrm{bp}$ & 0.8 & 1.5 & 3.3 & 2.1 & 3.2 & 10.4 \\
\hline & $6701 \mathrm{bp}$ & 0.7 & 1.1 & 2.1 & 1.4 & 4.6 & 10.1 \\
\hline & 8786 bp & 1.0 & 1.4 & 2.0 & 1.6 & 3.9 & 18.1 \\
\hline \multirow[t]{4}{*}{ Agilent Protein 80} & $5-80 \mathrm{kDa}$ & \multicolumn{3}{|c|}{3 (10 Accuracy) } & \multicolumn{3}{|c|}{20 (Accuracy not provided) } \\
\hline & 24.58 kDa (BamHI) & 0.2 & 0.4 & 2.1 & 12.6 & 20.0 & 28.3 \\
\hline & $61.23 \mathrm{kDa}(\mathrm{BSA})$ & 0.2 & 0.3 & 1.0 & 10.8 & 17.8 & 30.8 \\
\hline & $79.80 \mathrm{kDa}(\mathrm{CIP})$ & 0.2 & 0.3 & 0.9 & 10.9 & 13.6 & 20.4 \\
\hline
\end{tabular}

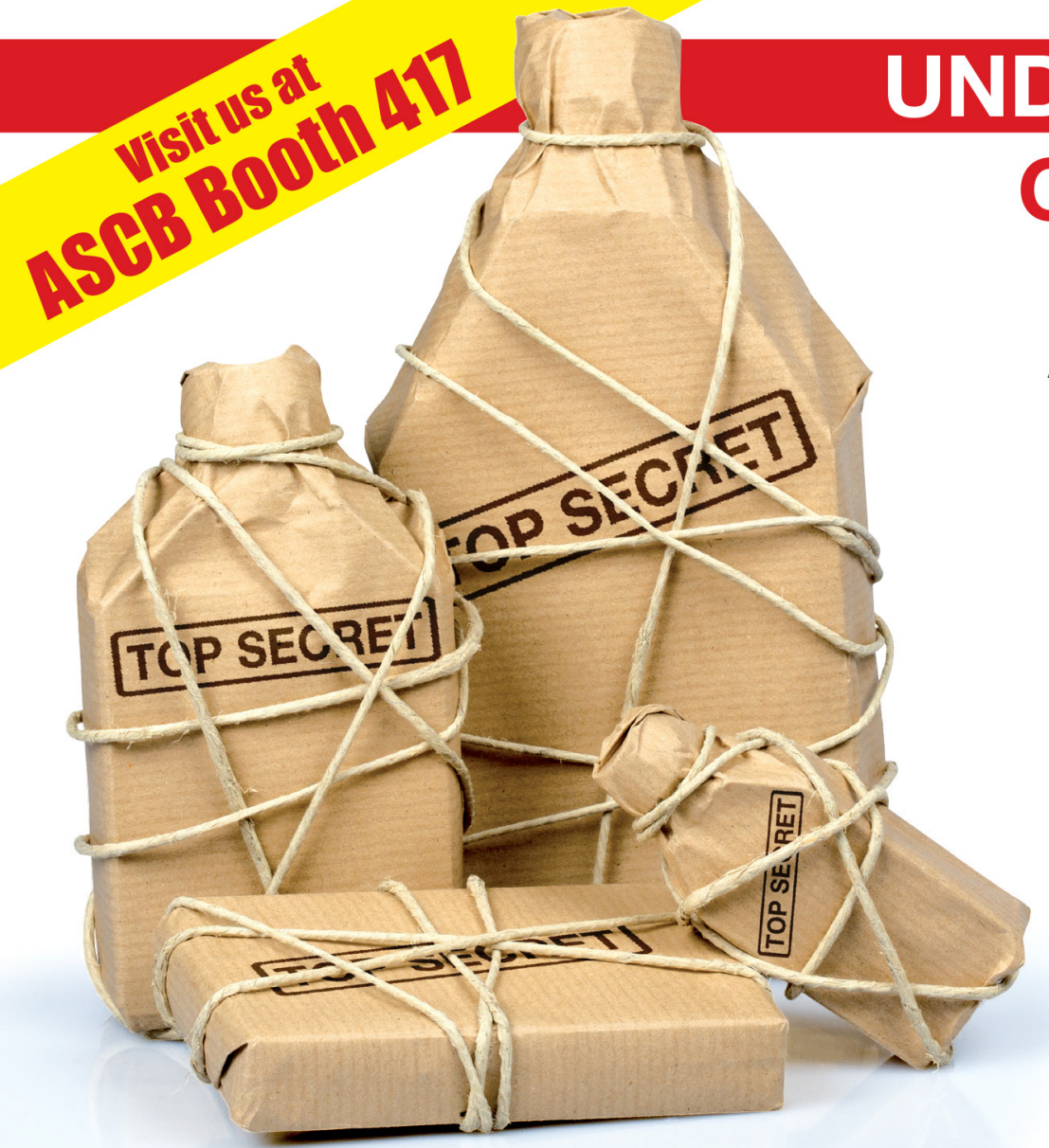

After 20 years, it was time for an update. We've painstakingly

- redesigned

- expanded

- optimized 


\section{Competing interests}

The authors declare no competing interests.

\section{References}

1. Brody, J.R. and S.E. Kern. 2004. History and principles of conductive media for standard DNA electrophoresis. Anal. Biochem. 333:1-13.

2. Mueller, O., K. Hahnenberger, M. Dittmann, H. Yee, R. Dubrow, R. Nagle, and D. Ilsley. 2000. A microfluidic system for high-speed reproducible DNA sizing and quantitation. Electrophoresis 21:128-134.

3. Chen, L. and J.C. Ren. 2004. High-throughput DNA analysis by microchip electrophoresis. Comb. Chem. High Throughput Screen. 7:29-43.

4. Blazek, V. and R.A. Caldwell. 2009. Comparison of SDS gel capillary electrophoresis with microfluidic lab-on-a-chip technology to quantify relative amounts of $7 \mathrm{~S}$ and $11 \mathrm{~S}$ proteins from 20 soybean cultivars. Int. J. Food Sci. Technol. 44:2127-2134.

5. Chim, W. and P.C.H. Li. 2012. Repeated capillary electrophoresis separations conducted on a commercial DNA chip. Anal. Methods 4:864-868.

6. Nakabayashi, H., K. Taketa, K. Miyano, T. Yamane, and J. Sato. 1982. Growth of Human Hepatoma Cell Lines with Differentiated Functions in Chemically Defined Medium. Cancer Res. 42:3858-3863.

Received 08 August 2014; accepted 14 October 2014.

Address correspondence to Steven J. Karpowicz, Department of Biology, University of Central Oklahoma, Edmond, OK. E-mail: skarpowicz@uco.edu

To purchase reprints of this article, contact:

biotechniques@fosterprinting.com

\section{Safe \&t Secure Weighing}

\section{Secura $^{\circledR}$}

\section{Reduce Your risk with integrated and intelligent protection systems.}

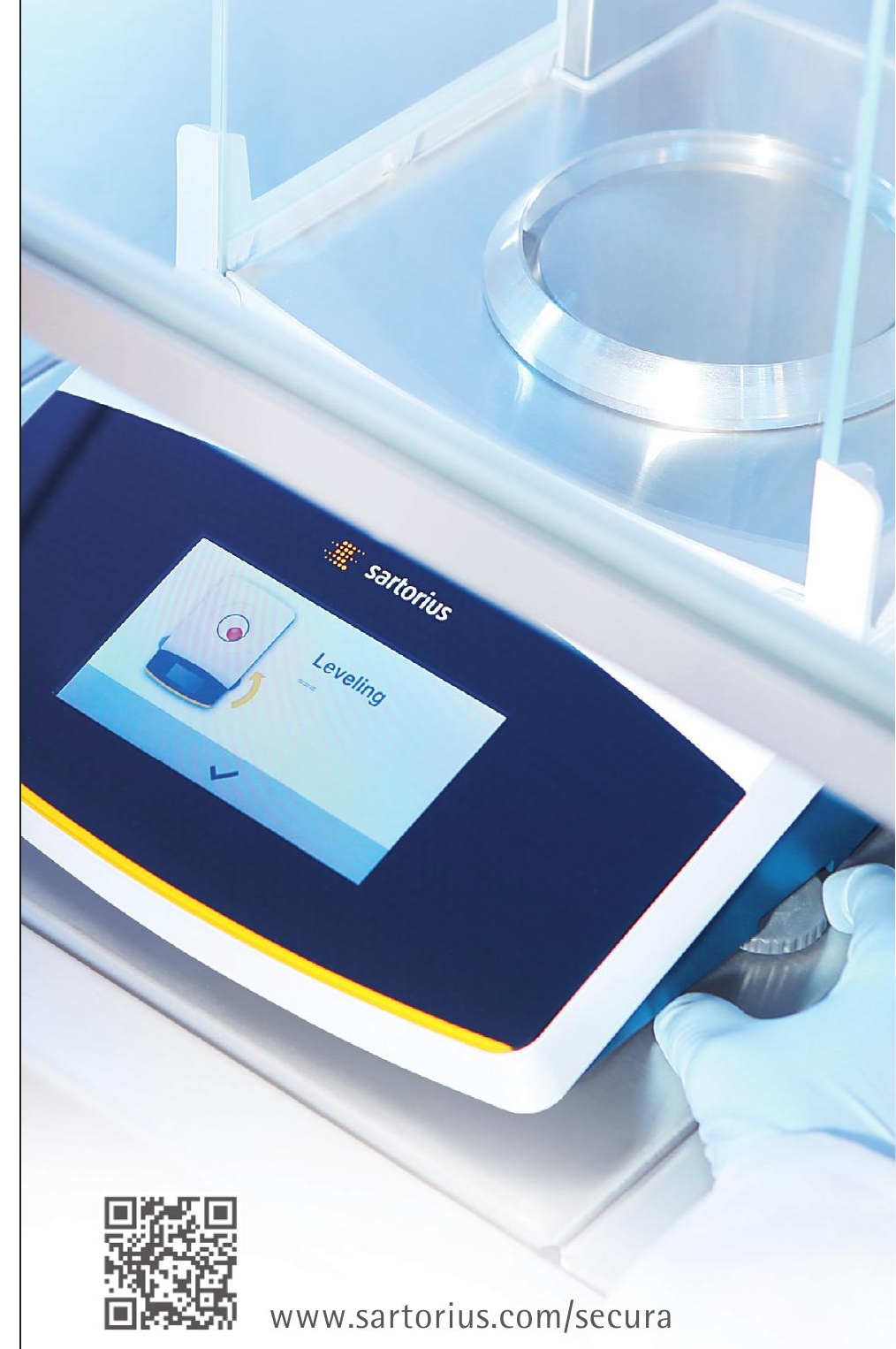

